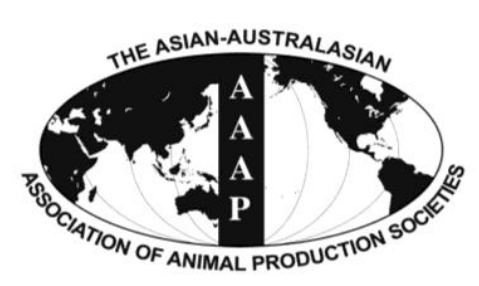

\title{
Genome-wide DNA Methylation Profiles of Small Intestine and Liver in Fast-growing and Slow-growing Weaning Piglets
}

\author{
Woori Kwak ${ }^{1,2}$, Jin-nam Kim ${ }^{1}$, Daewon Kim ${ }^{1}$, Jin Su Hong ${ }^{3}$, Jae Hark Jeong ${ }^{3}$, \\ Heebal Kim ${ }^{1,2,3}$, Seoae Cho ${ }^{1} *$, and Yoo Yong $\mathrm{Kim}^{3}{ }^{3} *$ \\ ${ }^{1}$ C\&K Genomics, Seoul 151-919, Korea
}

\begin{abstract}
Although growth rate is one of the main economic traits of concern in pig production, there is limited knowledge on its epigenetic regulation, such as DNA methylation. In this study, we conducted methyl-CpG binding domain protein-enriched genome sequencing (MBD-seq) to compare genome-wide DNA methylation profile of small intestine and liver tissue between fast- and slowgrowing weaning piglets. The genome-wide methylation pattern between the two different growing groups showed similar proportion of CpG (regions of DNA where a cytosine nucleotide occurs next to a guanine nucleotide in the linear sequence) coverage, genomic regions, and gene regions. Differentially methylated regions and genes were also identified for downstream analysis. In canonical pathway analysis using differentially methylated genes, pathways (triacylglycerol pathway, some cell cycle related pathways, and insulin receptor signaling pathway) expected to be related to growth rate were enriched in the two organ tissues. Differentially methylated genes were also organized in gene networks related to the cellular development, growth, and carbohydrate metabolism. Even though further study is required, the result of this study may contribute to the understanding of epigenetic regulation in pig growth. (Key Words: DNA Methylation, MBD-seq, Epigenetic Profile, Weaning Piglet, Genome-wide Methylation Profile)
\end{abstract}

\section{INTRODUCTION}

Pigs are an important economic animal and their growth rate is one of the main concerns of farmers, companies and scientists. Growth rate is known to be affected by the interaction of genetic and environmental factors in many animals including pigs (Hazel et al., 1943; Martorell et al., 1977; Bourdon and Brinks, 1982). In order to identify the genetic factors that underlie growth rate in pigs, many genome-wide association studies have been conducted. A number of different genes, variants and genetic regions

\footnotetext{
* Corresponding Authors: Seoae Cho. Tel: +82-2-876-8820, Fax: +82-2-876-8827, E-mail: seoae@cnkgenomics.com / Yoo Yong Kim. Tel: +82-2-880-4801, Fax: +82-2-878-5839, E-mail: yooykim@snu.ac.kr

${ }^{2}$ Interdisciplinary Program in Bioinformatics, Seoul National University, Seoul 151-747, Korea.

${ }^{3}$ Department of Agricultural Biotechnology, Seoul National University, Seoul 151-921, Korea.

Submitted Apr. 24, 2014; Revised Jun. 30, 2014; Accepted Aug. 15, 2014
}

were reported to have important effects on the growth rate (Lo et al., 1992; Mrode and Kennedy, 1993; Andersson et al., 1994; Estelle et al., 2008; Rubin et al., 2012).

While the genetic information based on the genome sequence can provide some insights, it has not been able to fully explain into the correlation between genome sequences and phenotypic traits. In light of this problem, epigenetic studies such as the ENCODE (encyclopedia of DNA element) project (The ENCODE Project Consortium, 2004; The ENCODE Project Consortium, 2011) have received attention because many phenotypic traits including growth rate could be influenced by epigenetic factors such as DNA methylation. Previous studies have shown that DNA methylation can affect gene regulation, phenotypic trait, genomic imprinting, and disease development ( $\mathrm{Li}$ et al., 1993; Siegfried et al., 1999; Bird, 2002; Dolinoy, 2008). In spite of the importance of DNA methylation, only a small number of studies have been conducted to identify genomewide methylation profiles of economic animals ( $\mathrm{Li}$ et al., 2011; Walker, 2012; Hu et al., 2013; Su et al., 2014). In 
particular, there has yet to be a study on genome-wide methylation and its relationship to economic traits such as growth rate in pigs.

In this study, we conducted methyl-CpG binding domain protein-enriched genome sequencing (MBD-seq) and bioinformatics analysis to assay the genome-wide DNA methylation pattern in the small intestine and liver samples of 21 day old weaning piglets with different growth rates. These two organs are important for digestion and absorption of nutrients. Besides, these organs undergo morphological changes and functional maturation during the postnatal period (Xu, 1996). For example, profound growth of organ tissue and the epithelial modification including the loss of ability of absorbing macromolecules occur in small intestine. The gene expression in liver is involved in metabolizing nutrients and generating growth factors like (Insulin-like growth factor $1(I G F-1)$. By comparing the genome-wide methylation pattern of two groups exhibiting different growth performances, we identified differentially methylated regions (DMRs) and genes related with these regions. Pathway and network analysis of these differentially methylated genes identified a number of candidate genes which may affect growth performance in pigs. This is the first study for the genome-wide DNA methylation distribution of weaning piglets using next generation sequencing and the results of this study may contribute to the improvement of epigenetic understanding of growth rate in pigs.

\section{MATERIAL AND METHODS}

\section{Animals}

Samples used in this study were a multicross hybrid breed of Yorkshire, Landrace, and Duroc. From three sows, the pigs with the highest and lowest average daily gain were selected 21 days after birth totaling six pigs. The average weight of the fast growing and slow growing pigs were 8.15 $\mathrm{kg}$ and $5.53 \mathrm{~kg}$ respectively. Liver and small intestine tissues were sampled from each pig for sequencing.

\section{Sequencing}

Before a genomic DNA library construction, methylated DNA fragments were captured using the Methycap kit (Diagenode, Denville, NJ, USA). TruSeqTM DNA Sample Preparation Kit (Illumina, San Diego, CA, USA) was used following the manufacturer's protocol. To generate raw data for each sample library, 51 cycle single-end sequencing using Hiseq 2000 was carried out. To obtain enough read for analysis, three samples were multiplexed within one lane of Hiseq 2000 sequencing lanes.

\section{Bioinformatics analysis}

FastQC (Andrews, 2010) was used to check the quality of the raw reads. Read bases with unnecessary sequences (adaptor, index, primer sequence of illumina platform) or low quality scores (under Q20 of phred scale) were filtered using Trimmomatic (Bolger et al., 2014). Filtered read were aligned to the pig reference genome (Sus scrofa 10.2, susScr3) using Bowtie2 (Langmead and Salzberg, 2012) with the default option parameters. Removal of duplicated read from polymerase chain reaction and alignment information sorting were conducted using Picard tools (http://picard.sourceforge.net) and Samtools (Li et al., 2009). To identify the DMR, methylated DNA immunoprecipitation sequencing data analysis (MEDIPS) (Chavez et al., 2013) and BSgenome (Pages, 2009) libraries of pig were used. The parameter setting of MEDIPS is as follows: uniq $=$ TRUE, extend $=300$, shift $=0$, window size $=100$. EdgeR method was used to identify DMR regions and false discovery rate (FDR) was used for multiple test correction. Regions shown to be significant (FDR <0.05) were used for downstream analysis. After identifying genomic windows that showed significant differential coverage between conditions, neighboring significant windows were merged into a large continuous region. The DMR regions were annotated using Peak analyzer v1.4 (Salmon-Divon et al., 2010) with three options: overlap, nearest downstream gene, and nearest transcription starting site). To calculate the proportion of uniquely mapped bases, various information (CpGs, repeats, genes) was downloaded from University of California, Santa Cruz (UCSC) table browser (Meyer et al., 2013). Canonical pathway and network analyses were conducted using Ingenuity Pathway Analysis (http://www.ingenuity.com, Fisher's exact test $\mathrm{p}<0.05)$.

\section{RESULTS}

\section{Genome-wide methylation profile and differentially methylated regions}

Produced and trimmed raw read data information from liver and small intestine samples of six pigs is described in Supplementary Table 1 . The average alignment rate of the 12 samples was $71.28 \%$, and on average $36.08 \%$ of reads was uniquely mapped to the reference genome. The details of the mapping rate of 12 samples are described in Table 1. The Pearson's correlation values of all samples were close to one in a saturation analysis using MEDIPs and the read coverage was enough to conduct downstream analysis. The saturation plots of the 12 samples are shown in Supplementary Figure 1. Supplementary Figure 2 shows the proportion of covered $\mathrm{CpG}$ region using pie chart. The covered $\mathrm{CpG}$ pattern for samples of the same organ showed similar patterns between high and low average daily gain groups. The sequence pattern coverage histogram (Supplementary Figure 3) also showed similar shape 
Table 1. Summary of read alignment rate of each sample using bowtie2 with default option (\%)

\begin{tabular}{lcccccc}
\hline & Intestine_high1 & Intestine_high2 & Intestine_high3 & Intestine_low1 & Intestine_low2 & Intestine_low3 \\
\hline Intestine & & & & & & \\
$\quad$ Overall alignment rate & 71.02 & 73.65 & 75.50 & 71.33 & 66.50 & 72.00 \\
Aligned exactly 1 time & 32.33 & 40.75 & 35.71 & 38.28 & 31.67 & 33.92 \\
Aligned over 1 time & 38.69 & 32.89 & 39.78 & 33.28 & 34.83 & 38.08 \\
$\quad$ Unaligned & 28.98 & 26.35 & 24.50 & 28.67 & 33.50 & 28.00 \\
Liver & & & & & \\
$\quad$ Overall alignment rate & 75.11 & 73.75 & 71.05 & 63.06 & 68.71 & 73.74 \\
Aligned exactly 1 time & 40.00 & 37.46 & 36.33 & 33.97 & 37.31 & 35.31 \\
Aligned over 1 time & 35.10 & 36.30 & 34.72 & 29.10 & 31.40 & 38.43 \\
$\quad$ Unaligned & 24.89 & 26.25 & 28.95 & 36.94 & 31.29 & 26.26 \\
\hline
\end{tabular}

between the two groups. On average, $0.27 \%$ of mapped reads did not cover any $\mathrm{CpG}$ region. Also, average enrichment score was 4.515 in the small intestine samples and 4.296 in the liver samples. This shows that MBD-seq used in this study properly captured the methylated DNA. Supplementary Table 2 shows the details of the enrichment score for each sample. The pairwise correlation plots of the genome wide coverage profiles between all samples used in this study were summarized in Supplementary Figure 4. Same as the CpG coverage pattern, there was no distinct difference between high and low average daily gain groups. Uniquely mapped based were categorized by regions of the genome (gene, $\mathrm{CpGs}$, repeats and others) and regions of the gene (exon, intron, 5'-untranslated region [UTR], 3'-UTR, upstream-2k and downstream-2K). Figure 1 shows the proportion of uniquely mapped bases for each sample. Most of the bases were located in repeat regions $(45.74 \%)$ and gene regions $(31.77 \%)$. On average $\mathrm{CpG}$ island regions
(2.54\%) made up a smaller proportion in comparison to other regions. Within the gene region, the average proportion of intron region was $84.50 \%$. Upstream $\_2 \mathrm{~K}$ region and downstream_2K region of genes were 5.92\% and $6.10 \%$, respectively. The sum proportion of Exon and UTR region was $3.76 \%$ on average. Same as the coverage of CpGs using pie chart and histogram, there was no distinct difference in the proportion of genome-wide methylation profile based on the genomic regions between two groups. After binomial test using MEDIPs, the number of identified and merged DMR in high average daily gain group was 30 (5 down, 25 up) in the small intestine tissue and 258 ( 3 down, 255 up) in the liver tissue compared to the low average daily gain group.

\section{Differentially methylated region annotation and pathway, network analysis}

Annotation results of DMR using peak analyzer with

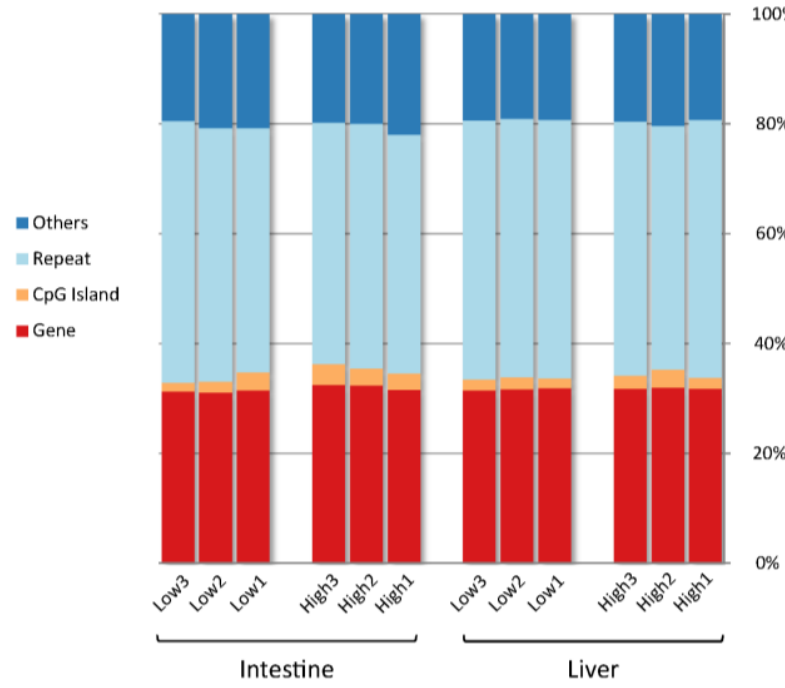

(a)

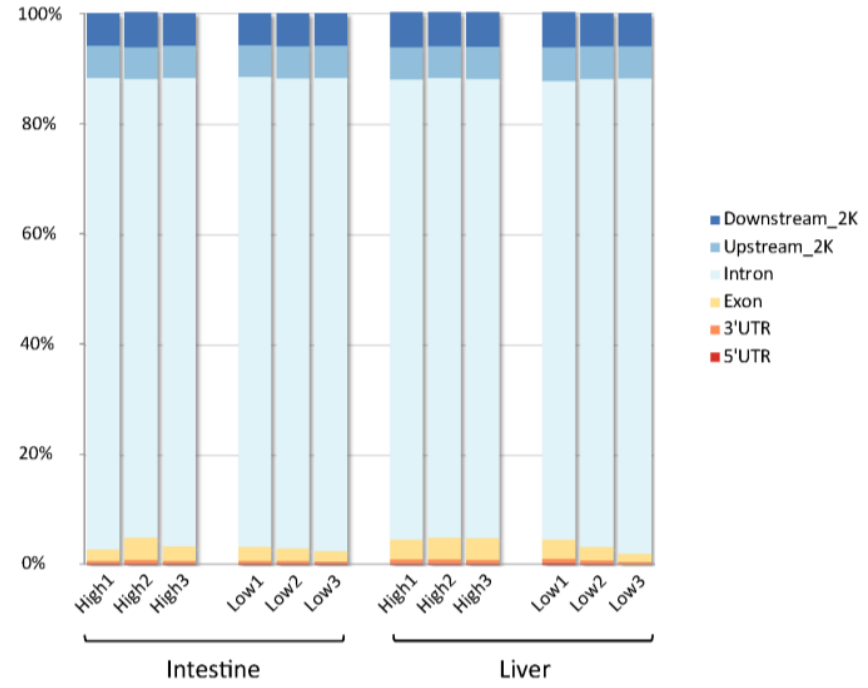

(b)

Figure 1. Genomic distribution of the uniquely mapped bases in two organ. (a) All uniquely mapped bases were classified into four type: bases uniquely mapped to genes (red), $\mathrm{CpG}$ Island (orange), repeats (light blue), others (blue). (b) Uniquely mapped bases in gene region were classified into 6 regions: bases uniquely mapped to 5'-UTR (red), 3'-UTR (orange), Exon (yellow), Intron (sky blue), Upstream_2K (light blue), Downstream_2K (Blue). 
a
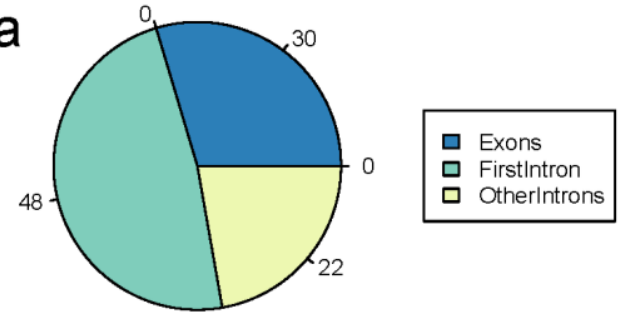

Intestine

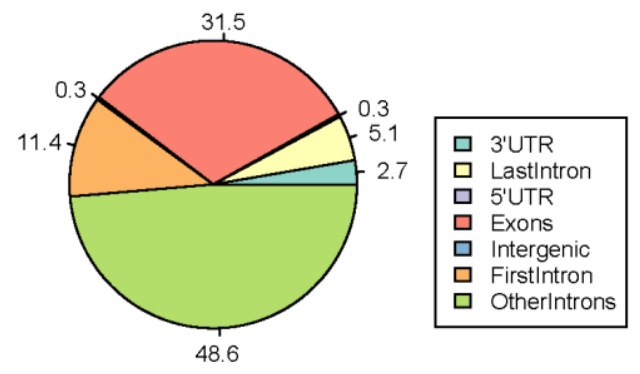

Liver b
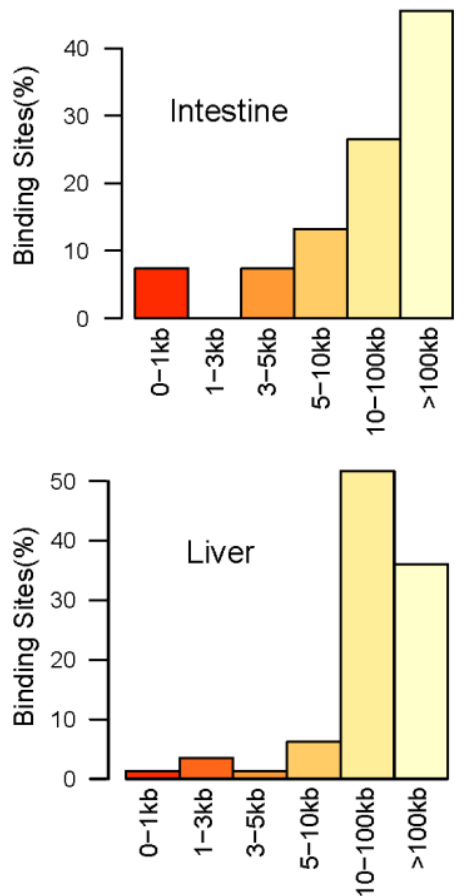

Figure 2. Annotation result of differentially methylated genes using Peak Analyzer. (a) Location and proportion of differentially methylated genes in two samples. (b) Distance distribution of nearest downstream genes from differentially methylated regions.

the Ensembl genome information is shown in (Figure 2). In the small intestine, $30 \%$ of DMR was located in exon region and $70 \%$ of DMR was located in intron region. In liver, $3 \%, 31.5 \%$, and $64.5 \%$ of DMR were located in UTR, exon and intron region, respectively. Distribution of the distances to the nearest downstream gene from DMR showed that most of DMRs were more than $5 \mathrm{~kb}$ from the downstream genes. The list of genes that overlapped with
DMRs, which were used in pathway and network analysis, is summarized in Table 2 and the full differentially methylated genes are listed in supplementary Table 3. Genes of nearest downstream gene and nearest transcription starting site are described in the supplementary Table 4.

Figure 3 shows the enrichment canonical pathway related to growth and cell cycle using ingenuity pathway analysis in the small intestine and liver tissues. The first

Table 2. Differentially methylated genes list using MEDIPs and peak analyzer (FDR <0.05)

\begin{tabular}{llll}
\hline Organ & \multicolumn{1}{c}{ Ensembl gene ID } & \multicolumn{1}{c}{ Gene symbol } & \multicolumn{1}{c}{ Gene description } \\
\hline Small intestine & ENSSSCG00000012939 & BRMS1-201 & Breast cancer metastasis suppressor 1 \\
& ENSSSCG00000013658 & SIPR2-201 & Sphingosine-1-phosphate receptor 2 \\
& ENSSSCG00000026161 & E2F6-201 & E2F transcription factor 6 \\
& ENSSSCG00000001025 & DSP-201 & Desmoplakin \\
& ENSSSCG00000014802 & NUMA1-201 & Nuclear mitotic apparatus protein 1 \\
& ENSSSCG00000016912 & PPAP2A-201 & Phosphatidic acid phosphatase type 2A \\
LNiver & ENSSSCG00000004012 & THBS2-201 & Thrombospondin 2 \\
& ENSSSCG00000005719 & $R A P G E F 1-201$ & Rap guanine nucleotide exchange factor (GEF) 1 \\
& ENSSSCG00000007602 & BAIAP2L1-201 & BAI1-associated protein 2-like 1 \\
& ENSSSCG00000008040 & $T S C 2-201$ & Tuberous sclerosis 2 \\
& ENSSSCG00000010756 & RTN4R-201 & Reticulon 4 receptor \\
& ENSSSCG00000011241 & DCLK3-201 & O-6-methylguanine-DNA methyltransferase \\
& ENSSSCG00000014811 & $I N P P L 1-201$ & Doublecortin-like kinase 3 \\
& ENSSSCG00000023677 & $C H M P 1 A-201$ & Inositol polyphosphate phosphatase-like 1 \\
& ENSSSCG00000026412 & $P L X N A 2-201$ & Plexin A2 \\
\hline
\end{tabular}

MEDIPs, methylated DNA immunoprecipitation sequencing data analysis; FDR, false discovery rate. 

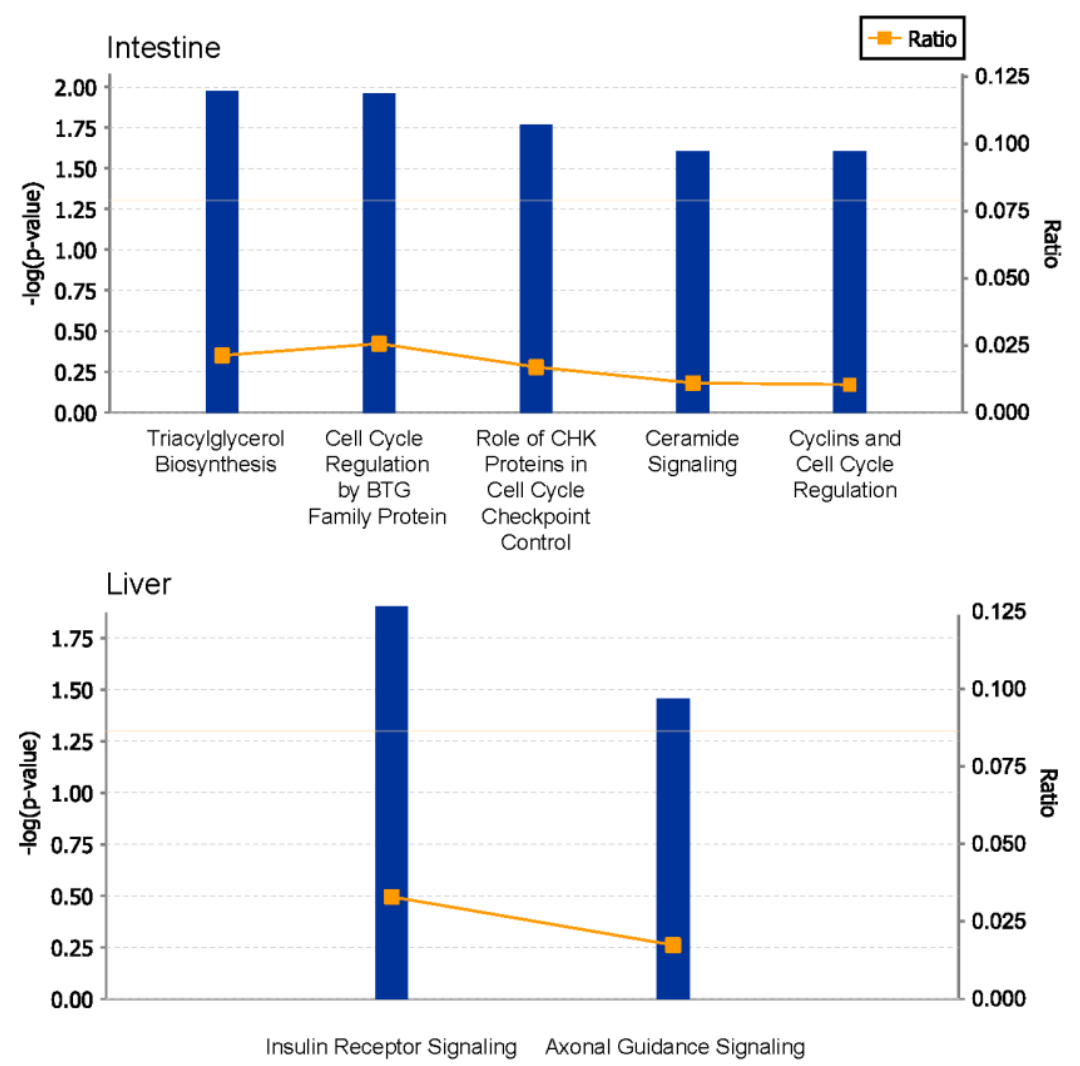

Figure 3. Enriched canonical pathways of differentially methylated genes using ingenuity pathway analysis (IPA). P-value of Fisher's exact test for enriched pathway, ratio: proportion of genes in the pathway, gray line: cut off line for transformed p-value 0.05 .

enriched canonical pathway of small intestine was the triacylglycerol biosynthesis pathway. And there were four enriched pathways related with cell cycle and cell growth (cell cycle regulation by B-cell translocation gene family protein, role of checkpoint kinase proteins in cell cycle checkpoint control, cyclins and cell cycle regulation, ceramide signaling). Phosphatidic acid phosphatase type 2A (PPAP2A), E2F6, and S1Pr2 genes were related to the enriched pathway. In liver, insulin receptor signaling and axonal guidance signaling pathway

were enriched. RAPGEF1, TSC2, INPPL1, BAIAP2, $P L X N A 2, C H M P 1 A$, and RTN4R were related to the two pathway enriched in liver.

Figure 4 shows the networks of genes overlapped with DMR in the two organs. In the small intestine, all genes (BRMS1, DSP, E2F6, NUMA1, and PPAP2A) except $S 1 P R 2$ were directly connected with ubiquitin $\mathrm{C}$ to form a network concerned with cancer, cell to cell signaling and a

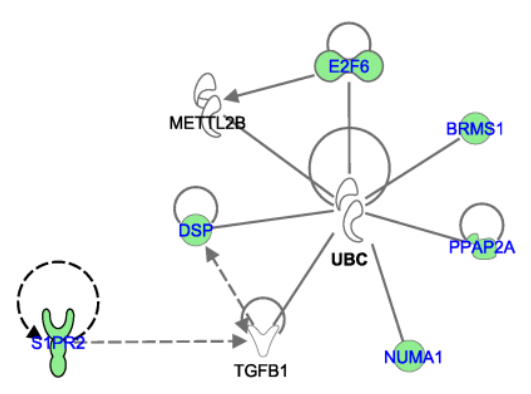

$<$ Intestine $>$ b
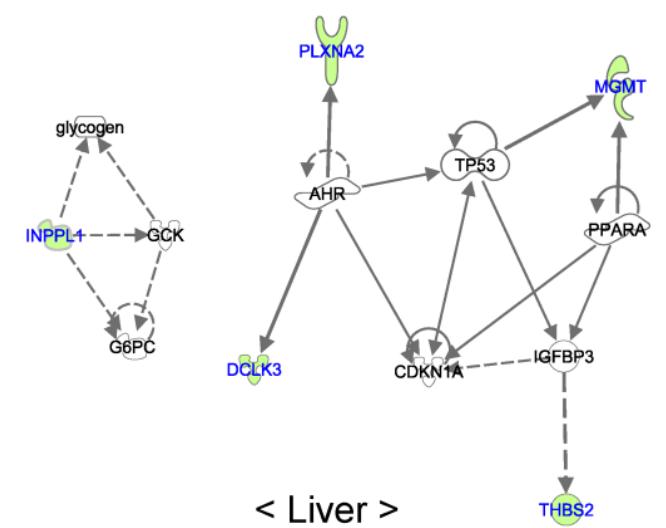

Figure 4. Identified gene networks using differentially methylated genes. Networks of potentially interacting proteins were placed into node. Nodes with green color indicate the identified differentially methylated genes in this study. 
interaction and cellular growth and proliferation. Seven genes (DCLK3, MGMT, PLXNA2, THBS2, TSPAN4, ILPPL1, and TSC2) overlapping with DMR regions the liver were associated with four networks. Among these networks, two networks were related to cellular growth and carbohydrate metabolism.

\section{DISCUSSION}

\section{Methylation profiles in small intestine and liver}

This is the first study to compare the genome-wide methylation profile of two organ tissues (small intestine and liver) between two groups of weaning period piglets, which show differential performance in growth. Small intestine and liver are important organs for piglets that absorb and metabolize the nutrition in breast milk. As it is well known that DNA methylation of promoter region and gene body region can affect the gene expression via chromatin structure changes or transcription efficiency (Lorincz et al., 2004; Klose and Bird, 2006; Suzuki and Bird, 2008), the object of this study was to compare the genome-wide methylation pattern and identify methylated genes that could affect the growth rate of piglets. Bases of uniquely mapped reads in our study were enriched in the repeat and gene regions and this was similar to the results of a previous study on chickens ( $\mathrm{Li}$ et al., 2011; Hu et al., 2013). However, the intron region comprised a large proportion of methylated genes $(>80 \%)$ compared to the other analysis. There was no big difference in the DNA methylation pattern (CpGs coverage and genomic regions) between two groups using enriched read location. A small number of differentially methylated genes were identified in the small intestine sample and a higher number of genes were methylated in the liver compared to the small intestine. The small intestine sampled showed no DMR located in UTR and only a small proportion of DMR of the liver were located in UTR. The distances of DMRs from the nearest downstream genes and transcription starting site under $1 \mathrm{~kb}$ was a small proportion of the overall distribution. This showed that the different epigenetical regulation between two groups may come from chromatin structure change, not from repress regulation in promoter regions.

\section{Potential genes and pathways related to the piglet growth rate}

Small intestine is the most important organ for piglet's absorption of nutrients. The breast milk which provide nutrients for the piglets has a high fat content. This fat in the breast milk is broken down by lipase and bile into monoacylgylcerol and free fatty acid. In these forms, it is absorbed into enterocytes, which is then rebuilt into tryacylglcerol and secreted as chylomicron before being used by the body. Enriched pathways and related genes in the small intestine were closely related to cell cycle regulation and lipid metabolism. Sphingosine-1-phosphate receptor $2(S 1 P R 2)$ is a member of $G$ protein-coupled receptor, and it is related to the sphingosine-1-phosphateinduced cell proliferation (An et al., 2000; Adada et al., 2013). E2F transcription factor 6 (E2F6) is a member of transcription factors that play a crucial role in the cell cycle control (Trimarchi et al., 2001). The protein of nuclear mitotic apparatus protein 1 (NUMAl) gene interacts with microtubules and organizes the mitotic spindle during cell division (Purohit et al., 1999). These genes are thought to be associated with the formation of villi of newborn, and villi play an important role in absorption of nutrients by expanding the surface of small intestine. PPAP $2 A$ is an integral membrane glycoprotein that conducts hydrolysis and uptake of lipids from extracellular space (Roberts et al., 1998). Therefore, these differentially methylated genes and related pathways are associated with digestion and absorption of breast milk of which a major nutrient is fat. This different nutrient absorption is thus thought to be related with the different growth rate of piglet before weaning.

Pathway analysis of the liver, which plays a key role in lipid and energy metabolism, showed that insulin receptor signaling and axonal guidance signaling pathway were enriched. Differentially methylated genes (INPPL1, PLXNA2, MGMT, DCLK3, and THBS2) formed networks such as carbohydrate metabolism, cellular growth and proliferation. Inositol polyphosphate phosphatase-like 1 (INPPL1) encodes an $\mathrm{SH} 2$-containing 5'-inositol phosphatase that is associated with the regulation of insulin function, and the functions of the liver are primarily controlled by the hormone insulin (Saltiel and Kahn, 2001; Fritsche et al., 2008). Previous study using INPPLI knockout mouse showed that the absence of INPPL1 confers the high resistance to the weight gain from high-fat diet (Sleeman et al., 2005). Hence, INPPL1 and enriched pathways related with the differentially methylated genes are thought to be closely related to the weight gain and growth rate of piglets.

Even though further study is required to identify the epigenetic effect of these genes, results of analysis of pathways and networks on growth rate of piglets may provide some clues to the epigenetic mechanism of piglet growth rate.

\section{ACKNOWLEDGMENTS}

This research was supported by the Korea Institute of Planning and Evaluation for Technology in Food, Agriculture, Forestry and Fisheries (iPET), the Advanced 
Production Technology Development Program, and the Ministry of Agriculture, Food and Rural Affairs.

\section{REFERENCES}

Adada, M., D. Canals, Y. A. Hannun, and L. M. Obeid. 2013. Sphingosine-1-phosphate receptor 2. FEBS J. 280:6354-6366.

An, S., Y. Zheng, and T. Bleu. 2000. Sphingosine 1-phosphateinduced cell proliferation, survival, and related signaling events mediated by $G$ protein-coupled receptors Edg3 and Edg5. J. Biol. Chem. 275:288-296.

Andersson, L., C. S. Haley, H. Ellegren, S. A. Knott, M. Johansson, K. Andersson, L. Andersson-Eklund, I. Edfors-Lilja, M. Fredholm, and I. Hansson. 1994. Genetic mapping of quantitative trait loci for growth and fatness in pigs. Science 263:1771-1774.

Andrews, S. 2010. FastQC: A quality control tool for high throughput sequence data. Reference Source. http://www.bioinformatics.babraham.ac.uk/projects/fastqc/ Accessed Month, Date, Year.

Bird, A. 2002. DNA methylation patterns and epigenetic memory. Genes Dev. 16:6-21.

Birney, E., J. A. Stamatoyannopoulos, A. Dutta, R. Guigó, T. R. Gingeras, E. H. Margulies, Z. Weng, M. Snyder, E. T. Dermitzakis, and R. E. Thurman. 2007. Identification and analysis of functional elements in $1 \%$ of the human genome by the ENCODE pilot project. Nature 447:799-816.

Bolger, A. M., M. Lohse, and B. Usadel. 2014. Trimmomatic: A flexible trimmer for Illumina Sequence Data. Bioinformatics doi:10.1093/bioinformatics/btu170

Bourdon, R. and J. Brinks. 1982. Genetic, environmental and phenotypic relationships among gestation length, birth weight, growth traits and age at first calving in beef cattle. J. Anim. Sci. 55:543-553.

Chavez, L., M. Lienhard, and J. Dietrich. 2013. MEDIPS: (MeD)IP-seq data analysis. R package version 1.14.0.

Dolinoy, D. C. 2008. The agouti mouse model: an epigenetic biosensor for nutritional and environmental alterations on the fetal epigenome. Nutr. Rev. 66:S7-S11.

Estelle, J., F. Gil, J. Vazquez, R. Latorre, G. Ramirez, M. C. Barragan, J. M. Folch, J. L. Noguera, M. A. Toro, and M. Perez-Enciso. 2008. A quantitative trait locus genome scan for porcine muscle fiber traits reveals overdominance and epistasis. J. Anim. Sci. 86:3290-3299.

Fritsche, L., C. Weigert, H.-U. Haring, and R. Lehmann. 2008. How insulin receptor substrate proteins regulate the metabolic capacity of the liver -implications for health and disease. Curr. Med. Chem. 15:1316-1329.

Hazel, L. N., M. L. Baker, and C. F. Reinmiller. 1943. Genetic and environmental correlations between the growth rates of pigs at different ages. J. Anim. Sci. 2:118-128.

Hu, Y., H. Xu, Z. Li, X. Zheng, X. Jia, Q. Nie, and X. Zhang. 2013. Comparison of the genome-wide DNA methylation profiles between fast-growing and slow-growing broilers. PloS one 8(2):e56411.

Klose, R. J. and A. P. Bird. 2006. Genomic DNA methylation: the mark and its mediators. Trends Biochem. Sci. 31:89-97.

Langmead, B. and S. L. Salzberg. 2012. Fast gapped-read alignment with Bowtie 2. Nat. Methods 9:357-359.
Li, E., C. Beard, and R. Jaenisch. 1993. Role for DNA methylation in genomic imprinting. Nature 366:362-365.

Li, H., B. Handsaker, A. Wysoker, T. Fennell, J. Ruan, N. Homer, G. Marth, G. Abecasis, and R. Durbin. 2009. The sequence alignment/map format and SAMtools. Bioinformatics 25:20782079.

Li, Q., N. Li, X. Hu, J. Li, Z. Du, L. Chen, G. Yin, J. Duan, H. Zhang, Y. Zhao, J. Wang, and N. Li. 2011. Genome-wide mapping of DNA methylation in chicken. PLoS One 6(5):e19428.

Lo, L., D. McLaren, F. McKeith, R. Fernando, and J. Novakofski. 1992. Genetic analyses of growth, real-time ultrasound, carcass, and pork quality traits in Duroc and Landrace pigs: II. Heritabilities and correlations. J. Anim. Sci. 70:2387-2396.

Lorincz, M. C., D. R. Dickerson, M. Schmitt, and M. Groudine. 2004. Intragenic DNA methylation alters chromatin structure and elongation efficiency in mammalian cells. Nat. Struct. Mol. Biol. 11:1068-1075.

Martorell, R., C. Yarbrough, A. Lechtig, H. Delgado, and R. E. Klein. 1977. Genetic-environmental interactions in physical growth. Acta Paediatr. Scand. 66:579-584.

Meyer, L. R., A. S. Zweig, A. S. Hinrichs, D. Karolchik, R. M. Kuhn, M. Wong, C. A. Sloan, K. R. Rosenbloom, G. Roe, and B. Rhead et al. 2013. The UCSC Genome Browser database: extensions and updates 2013. Nucl. Acids Res. 41:D64-D69.

Mrode, R. and B. Kennedy. 1993. Genetic variation in measures of food efficiency in pigs and their genetic relationships with growth rate and backfat. Anim. Prod. 56:225-225.

Pages, H. 2009. BSgenome: Infrastructure for Biostrings-based genome data packages. $\mathrm{R}$ package version 1.32.0

Purohit, A., S. H. Tynan, R. Vallee, and S. J. Doxsey. 1999. Direct interaction of pericentrin with cytoplasmic dynein light intermediate chain contributes to mitotic spindle organization. J. Cell Biol. 147:481-492.

Roberts, R., V. A. Sciorra, and A. J. Morris. 1998. Human type 2 phosphatidic acid phosphohydrolases. Substrate specificity of the type $2 \mathrm{a}, 2 \mathrm{~b}$, and $2 \mathrm{c}$ enzymes and cell surface activity of the 2a isoform. J. Biol. Chem. 273:22059-22067.

Rubin, C.-J., H.-J. Megens, A. M. Barrio, K. Maqbool, S. Sayyab, D. Schwochow, C. Wang, Ö. Carlborg, P. Jern, and C. B. Jørgensen et al. 2012. Strong signatures of selection in the domestic pig genome. Proc. Natl. Acad. Sci. 109:19529-19536.

Salmon-Divon, M., H. Dvinge, K. Tammoja, and P. Bertone. 2010. PeakAnalyzer: genome-wide annotation of chromatin binding and modification loci. BMC Bioinformatics 11:415.

Saltiel, A. R. and C. R. Kahn. 2001. Insulin signalling and the regulation of glucose and lipid metabolism. Nature 414:799806.

Siegfried, Z., S. Eden, M. Mendelsohn, X. Feng, B.-Z. Tsuberi, and H. Cedar. 1999. DNA methylation represses transcription in vivo. Nat. Genet. 22:203-206.

Sleeman, M. W., K. E. Wortley, K.-M. V. Lai, L. C. Gowen, J. Kintner, W. O. Kline, K. Garcia, T. N. Stitt, G. D. Yancopoulos, S. J. Wiegand, and D. J. Glass. 2005. Absence of the lipid phosphatase SHIP2 confers resistance to dietary obesity. Nat. Med. 11:199-205.

Su, J., Y. Wang, X. Xing, J. Liu, and Y. Zhang. 2014. Genomewide analysis of DNA methylation in bovine placentas. BMC Genomics 15:12.

Suzuki, M. M. and A. Bird. 2008. DNA methylation landscapes: 
Provocative insights from epigenomics. Nat. Rev. Genet. Trimarchi, J. M., B. Fairchild, J. Wen, and J. A. Lees. 2001. The 9:465-476.

The ENCODE Project Consortium. 2004. The ENCODE (ENCyclopedia of DNA elements) project. Science 306:636640.

The ENCODE Project Consortium. 2011. A user's guide to the encyclopedia of DNA elements (ENCODE). PLoS Biol. 9(4):e1001046. E2F6 transcription factor is a component of the mammalian Bmi1-containing polycomb complex. Proc. Natl. Acad. Sci. 98:1519-1524.

Xu, R.-J. 1996. Development of the newborn GI tract and its relation to colostrum/milk intake: A review. Reprod. Fertil. Dev. 8:35-48. 